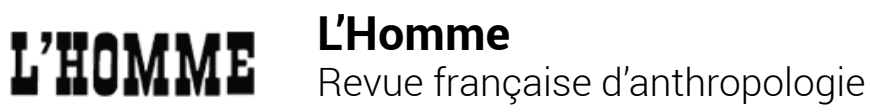

175-176 | juillet-septembre 2005

Vérités de la fiction

\section{Sandra J. T. M. Evers, Constructing History, Culture and Inegality. The Betsileo in the Extreme Southern Highlands of Madagascar \\ Leiden, Brill, 2002, 241 pages}

Sophie Blanchy

\section{OpenEdition}

Journals

Édition électronique

URL : http://journals.openedition.org//homme/2079

DOI : 10.4000/lhomme.2079

ISSN : 1953-8103

Éditeur

Éditions de l'EHESS

Édition imprimée

Date de publication : 15 octobre 2005

Pagination : $561-564$

ISBN : 2-7132-2035-1

ISSN : 0439-4216

\section{Référence électronique}

Sophie Blanchy, « Sandra J. T. M. Evers, Constructing History, Culture and Inegality. The Betsileo in the Extreme Southern Highlands of Madagascar », L'Homme [En ligne], 175-176 | juillet-septembre 2005, mis en ligne le 30 novembre 2006, consulté le 24 septembre 2020. URL : http://journals.openedition.org/ Ihomme/2079; DOI : https://doi.org/10.4000//homme.2079

Ce document a été généré automatiquement le 24 septembre 2020.

(c) École des hautes études en sciences sociales 


\section{Sandra J. T. M. Evers, Constructing History, Culture and Inegality. The Betsileo in the Extreme Southern Highlands of Madagascar}

Leiden, Brill, 2002, 241 pages

Sophie Blanchy

\section{RÉFÉRENCE}

Sandra J. T. M. Evers, Constructing History, Culture and Inegality. The Betsileo in the Extreme Southern Highlands of Madagascar, Leiden, Brill, 2002, 241 p., bibl., gloss., index (« African Social Studies Series » 4).

1 CETTE eXCELLENTE ÉTUDE menée dans un village du sud des Hautes Terres de Madagascar détaille les mécanismes de la construction de l'inégalité sociale, et plus précisément du statut d'esclave (andevo), en explorant les processus économiques et culturels qui les sous-tendent.

L'esclavage fut aboli à Madagascar en 1896, au début de la colonisation française, après l'affranchissement de certaines catégories d'esclaves sous la royauté merina. De nombreuses régions situées à la frontière des anciens ensembles politiques régionaux ne furent peuplées qu'à cette époque par les anciens esclaves, qui avaient ainsi l'opportunité de rompre avec leur passé servile et de devenir tompon-tany, « maîtres du sol », sur des terres inoccupées. Le statut de tompon-tany ne devient tangible qu'avec la construction d'un tombeau familial permanent, généralement consacré «tombeau ancestral» par le transfert d'un ancêtre pris dans le tombeau du lieu d'origine. L'absence de ces transferts, et d'autres indices, autorisent Sandra Evers à penser que les tompon-tany du village de Marovato où elle a enquêté sont eux-mêmes des descendants 
d'andevo, ayant migré depuis des villages fondés après l'abolition par ceux que l'on nommait olona vaovao (" personnes neuves ", anciens esclaves).

3 L'histoire du village fait apparaître plusieurs phases d'implantation. Les familles venues avant 1967 avaient conclu entre elles un pacte de nature politique concernant les terres entourant le village, pacte qui verrouillait le statut de tompon-tany: les nouveaux venus ne seraient jamais que des migrants (mpiavy), exclus de la propriété foncière. Les migrants installés au village s'avèrent relever de deux statuts bien distincts et strictement endogames : libres ou « blancs » (olompotsy) pour ceux qui peuvent prouver leur origine, c'est-à-dire l'existence d'un tombeau ancestral, esclaves (andevo) pour les autres, contraints de s'installer au sud-ouest du village, ce qui, compte tenu de la symbolique spatiale à Madagascar, les stigmatise encore davantage. Tous les migrants louent les terres qu'ils cultivent aux maittres du sol; ces derniers ne laissent aux andevo que les plus mauvaises, ce qui les enferme dans un cycle de dettes rendant leur dépendance économique sans issue.

4 L'auteur découvre peu à peu la réalité insoupçonnée du statut d'esclave à Marovato, et concentre sa recherche sur cette question : en quoi consiste ce statut et comment peutil être imposé, et surtout accepté, dans des formes aussi violentes et radicales? Elle se heurte à un évitement tant de la part des dominants dirigeant le village qui l'enjoignent de ne pas fréquenter les andevo, que de la part de ces derniers peu enclins à raconter l'histoire de leur sujétion. L'enquête, difficile, a demandé du courage et de la patience. Le contact de l'auteur avec des "personnes impures " (olona maloto) l'a obligée à se soumettre à un rituel de purification : "pur/impur » apparaît en fin de compte comme la dernière paire d'opposition qui structure les rapports sociaux hiérarchiques de cette société villageoise, séparant les andevo des autres, tous statuts confondus, nobles ou roturiers, propriétaires ou migrants, en se fondant sur des éléments culturels: existence d'ancêtres, possession de hasina (force de vie positive reçue des ancêtres), capacité à devenir ancêtre après la mort.

Les andevo de Marovato intériorisent le statut que les autres leur ont assigné, et leur groupe d'exclus ne développe aucun des modes de sociabilité des hommes libres: peu de cohésion familiale et sociale, aucun rituel. À l'inverse, le groupe des maîtres du sol, malgré l'absence de lignées patrilinéaires fortes, multiplient leurs liens sociaux par des alliances matrimoniales dans les réseaux régionaux, par le rituel de vaki-rà (alliance par le sang) et par le déplacement d'enfants entre maisonnées (fosterage). Les rituels donnent l'occasion à ces dominants d'établir, au cours de longs monologues, leur version de la réalité sur eux-mêmes et sur les autres. Ces discours formels, ainsi que les rumeurs que l'on fait courir dans le village, sont deux techniques de domination que maîtrise la seule famille non suspecte d'une origine andevo, rattachée à l'ancienne famille royale de cette région du Betsileo, et se déclarant donc andriana (noble). Cette appartenance crée un lien hiérarchique entre le village et une source de pouvoirs politique et rituel. Le groupe des maîtres du sol (tompon-tany) est également soumis au pouvoir de ses dirigeants, et à leur violence potentielle : des projets de mariage avec des andevo, qui menacent directement l'intégrité du groupe, provoquent de graves tensions entre père et fils. Les soupçons de magie et de sorcellerie traduisent les intentions de mort imputées aux parents ou aux voisins; l'empoisonnement, très redouté à Madagascar, est reconnu comme la cause de plusieurs morts survenues en plein conflit. Le statut que les tompon-tany assignent aux andevo dans le village leur permet de maintenir leur propre supériorité ; aussi, un conjoint qui se révèle être 
andevo doit-il être chassé. Si le couple a déjà des enfants, l'andevo impur est à peine toléré et sera exclu du tombeau familial. Dans le seul couple mixte dont Sandra Evers ait eu connaissance pendant longtemps, le mari, éleveur de bœufs, était venu rejoindre son oncle à Marovato après avoir été ruiné par un raid de voleurs dans son village, puis il avait épousé une andevo sans exigences sur la dot. Son oncle chef de famille s'arrangeait de la présence de l'andevo dans sa maisonnée en allant régulièrement se faire purifier auprès de la famille royale.

Cette ethnographie finement conduite sur la manière dont se construisent les relations hiérarchiques à Marovato nous instruit sur les processus mis en œuvre en d'autres lieux, aujourd'hui comme à d'autres époques, dans la société malgache. Ce sont essentiellement les rituels qui contribuent à établir une réalité partagée, intériorisée par tous, légitimant des conditions économiques qui perpétuent la dépendance. Entraînés dans un système de dettes toujours plus lourdes, les andevo de Marovato survivent grâce à des trafics illicites (alcool local, chanvre) qui confortent l'image qui leur est socialement assignée.

7 Revenue quatre ans plus tard pour un deuxième séjour de terrain, Sandra Evers constate que la catégorie des "gens purs » du village s'est renforcée d'un nouveau groupe, les salariés (mpikarama), principalement des alliés venant des familles des épouses des maîtres du sol (tompon-tany), se réclamant tous d'une origine libre. Ils constituent un groupe concurrent de celui des andevo sur le plan économique. L'opération, dirigée par un nouveau venu noble (andriana) adopté par le dirigeant du village grâce au rituel de vaki-rà (alliance de sang), consiste à augmenter le rendement des terres en les faisant travailler par ces parents pauvres, tout en favorisant le départ des andevo. Les "salariés ", jeunes gens célibataires logés dans un premier temps chez leurs parents employeurs, pouvaient être intégrés dans le statut de migrants en construisant une maison dans le village et en louant des terres, tout en restant liés à leur tombeau ancestral, ou bien devenir tompon-tany en participant au frais d'entretien du tombeau des parents, où ils auraient alors droit d'entrer. En réalité, la plupart d'entre eux se sont fait enfermer dans un système de dettes et de dépendance identique à celui des autres migrants, mais soigneusement dissimulé par l'usage du langage de la parenté (fihavanana), outil de domination supplémentaire.

8 La manipulation idéologique à laquelle se livrent les dirigeants du village est plus visible dans le cas des couples mixtes, comme en témoigne l'exemple suivant. Confronté à la révélation gênante de l'origine andevo d'une épouse dans sa propre famille, le dirigeant du village décide que cette femme n'aura pas accès au tombeau ancestral du mari et que ce dernier devra subir après sa mort une purification rituelle pour pouvoir y entrer. Quand l'ethnologue insiste pour savoir comment il avait pu ignorer jusque-là cet état de choses, il lui fait le reproche habituel : « Tu poses trop de questions ", laissant entendre que la construction de la hiérarchie sociale du village ne se fait pas mécaniquement par le seul jeu de critères objectifs, mais relève de la stratégie politique des dominants comme lui. De même, une femme âgée et divorcée, venue s'installer au village pour commencer une nouvelle vie, avait été contrainte de s'installer à l'ouest, ses prétentions d'être andriana de la région d'Ambositra n'ayant pas été reconnues. Plus tard sa fille, elle-même divorcée, vient la rejoindre puis se marie avec le fils du dirigeant $d u$ village. Celui-ci feint alors d'avoir obtenu des informations confirmant l'existence du tombeau ancestral andriana revendiquée par la 
mère, et reclasse cette famille désormais alliée à la sienne comme libre, blanche et pure.

9 Gens sans tombeaux, les andevo acceptent et intériorisent les discours tenus sur eux, y compris la théorie locale sur l'ontogenèse de chaque catégorie sociale. L'être humain, dit « être vivant » (olombelona) avant la mort et « ancêtre » (razana) après, est composé d'au moins deux instances spirituelles, l'ambiroa et le fanahy. L'ambiroa est le siège du hasina, une force de vie que tout le monde possède mais à des degrés très divers: les enfants en ont peu, les vieillards beaucoup. À la mort, tandis que le fanahy part vers la montagne des morts, Anbondrombe, l'ambiroa se joint dans le tombeau à celui des autres ancêtres, et leur hasina accumulé sert à protéger les descendants. Les andevo, dont l'ambiroa contient très peu de hasina, ne sont pas mis dans des tombeaux collectifs, mais, à Marovato du moins, enterrés n'importe où, à la sauvette et non sans honte de leur part (on comprendra pourquoi plus loin : incapables de produire du hasina, leurs morts non ancestralisés sont dangereux pour la communauté des vivants). Sandra Evers nous livre une ethnographie rare et impressionnante sur la violence du sort qui leur est fait quand meurt l'un d'entre eux, ici une toute jeune femme après la naissance de son premier enfant. Ce genre d'enterrement furtif était rapporté au XIX ${ }^{e}$ siècle par le R. P. Callet pour les andevo d'Imerina, près d'Antananarivo. Le corps de la jeune morte est porté, de nuit, à des heures de marche du village, et enterré sans un mot dans un trou recouvert de pierres. C'est que, pour la théorie dominante, l'ambiroa de ces gens sans tombeaux peut errer autour du village et y déverser son hasina devenu hery, une force dangereuse provoquant la maladie et la mort. Dans un système de représentations qui fait de l'ancestralisation un processus central, son absence laisse un creux où vont s'accumuler toutes les interprétations du mal. La nature même de l'état d'andevo, son impureté, sont liées à un faible niveau de hasina. Celui-ci est essentiellement dû à leur mise en sujétion, et leur impureté aux multiples tâches sales qu'ils assurent quotidiennement depuis des générations.

Dans certaines régions de Madagascar, une simple purification suffit à rendre à l'affranchi son potentiel de hasina. Sandra Evers se demande alors d'où vient la dureté et la radicalité des rapports entre catégories établis dans le village de Marovato, sachant précisément que ces tompon-tany ne sont rien d'autre que d'anciens andevo, exceptée une seule famille. En Imerina, les andevo ont acheté des terres et construit des tombeaux au début $\mathrm{du} \mathrm{xx}^{\mathrm{e}}$ siècle, et si la hiérarchie sociale existe toujours, elle s'exprime de manière plus feutrée. À Marovato, l'impureté des esclaves est irréversible, et la purification réservée à ceux qui les ont touchés. Aussi l'auteur émet l'hypothèse que les andevo de Marovato sont pour les tompon-tany la mémoire occultée de l'esclavage passé. Analysant la situation de ce village dans l'optique des sociétés frontalières développée par Igor Kopytoff pour l'Afrique, elle relit la manière dont ces premiers venus travaillent à construire leur statut de gens libres, en utilisant les concepts de la société d'où ils viennent, et où ils n'avaient pas la même position. Ils s'installent donc en érigeant des tombeaux puis en maintenant leurs bonnes relations avec leurs ancêtres par de multiples rituels préparés avec l'aide constante des ombiasa, et enfin en tissant des alliances. Dans ce travail intensif, les andevo ont un rôle à jouer : ils représentent en négatif ce que sont les tompon-tany. C'est ainsi qu'il faut comprendre, dans ce cas précis, l'irréversibilité de leur statut. L'auteur conclut que le maintien de l'esclavage par les maîtres du sol de Marovato n'est rien moins qu'une 
technique de survie. Loin d'être un système stable et intemporel, il s'agit d'un procès dynamique, placé ici dans un contexte matériel très difficile.

11 On retiendra la richesse de l'analyse de ce procès, qui éclaire les autres situations d'inégalité sociale ailleurs à Madagascar, situations toujours incluses dans des contextes variables, mais s'appuyant sur ce que Igor Kopytoff, dans sa théorie de la frontière, appelle la métropole, c'est-à-dire la communauté d'origine et sa culture. Les recompositions sociales, dans les villages "nouveaux» et dans les villes malgaches, jouent sur des rapports de domination, qui s'alimentent de valeurs culturelles dont les concepts de tombeaux, ancêtres et parenté constituent toujours les fondements. 